\title{
24th Annual Brain Impairment Conference
}

\section{The Kevin Walsh Encouragement Award for Honours or Masters Research}

was awarded to Sarah Farmer for the following presentation:

\section{Assessment of Premorbid Cognitive Ability in Patients with Schizophrenia}

\author{
Sarah Farmer ${ }^{1}$, Maria Hennessy ${ }^{1}$ and John Allan ${ }^{2}$ \\ ${ }^{1}$ School of Psychology, James Cook University, Townsville QLD \\ ${ }^{2}$ Psychiatry, Townsville General Hospital, Townsville QLD
}

\begin{abstract}
$\mathrm{T}$ The assessment of premorbid intelligence, or previous level of cognitive ability, has been examined extensively in relation to organic diseases of the brain, particularly Dementia of the Alzheimers Type. However, it is only recently that this area has been investigated in relation to patients diagnosed with schizophrenia. Numerous studies have found that vocabulary and word knowledge are relatively resistant to neurological damage, and correlate highly with general intelligence. To date, studies assessing premorbid intelligence in schizophrenia have generally used only one or two measures of previous ability. This paper examined intellectual functioning in patients with schizophrenia in comparison to a matched control group, using a number of purported measures of premorbid intelligence (AUSNART, CCRT, STW, WRAT3 Reading subtest, PPVT-3) as well as a measure of current cognitive functioning (WAIS-III). The PPVT-3 has not been used to assess premorbid cognitive ability thus far. No significant differences were found between groups for performances on the AUSNART, CCRT or WRAT3. These tests also had significant correlations with WAIS-III Full Scale IQ and Verbal IQ, thus supporting their use as 'hold' tests of premorbid IQ in an Australian population. This study also found further support for the validation of the AUSNART.
\end{abstract}

\section{The Luria Award for Doctoral Research was awarded to Tanya Lye} for the following presentation:

\section{MRI Correlates of "Normal" Brain Ageing in the Ninth and Tenth Decades of Life}

Tanya Lye ${ }^{1}$, D Grayson ${ }^{1}$, Hayley Bennett ${ }^{1}$, Olivier Piguet ${ }^{1}, \mathrm{H}_{\text {Creasey }}{ }^{1}$ and Tony Broe ${ }^{2}$

${ }^{1}$ Centre for Education and Research on Ageing, Concord Hospital, Sydney NSW

2 Prince of Wales Medical Institute, Sydney NSW

\begin{abstract}
$\mathrm{M}$ RI-based volumetry was employed to investigate whether hippocampal volumes were selectively correlated with memory performance in 102 community-dwelling individuals aged between 81 and 94 years. Participants were assessed on a variety of clinical and experimental instruments, as well as undergoing a neurological examination. An extensive medical history was obtained from an informant to confirm details of each participant's functional ability. Three measures of the hippocampus were compared to determine which measures were predictive of memory functioning: (1) hippocampal volumes adjusted by whole brain volumes; (2) hippocampal volumes adjusted by total intracranial volume; and (3) visual ratings of the hippocampus. All hippocampal measures were found to be significant predictors of delayed
\end{abstract}


recall of short stories, although whole brain adjusted hippocampal volumes and visual ratings of the hippocampus were the best predictors of memory functioning in this group. Hippocampal measures were generally not associated with performance in other cognitive domains. When the sample was reduced to include only the most cognitively healthy participants, the left hippocampal volume (whole brain adjusted) remained a significant predictor of retention of short stories. These findings suggest that hippocampal volumes are selectively correlated with memory functioning in healthy individuals in their ninth and tenth decade of life. 